\title{
Synthesis of the fungal natural product (-)-xylariamide A
}

Rohan A. Davis ${ }^{\mathrm{a}, *}$ and Michael Kotiw ${ }^{\mathrm{b}}$

${ }^{a}$ Chemical Biology Program, Eskitis Institute, Griffith University, Brisbane, QLD 4111, Australia

${ }^{\mathrm{b}}$ Department of Biological and Physical Sciences, University of Southern Queensland, Toowoomba, QLD 4351, Australia

* Corresponding author. Tel.: +61 73875 6043; fax: +61 73875 6001; e-mail: r.davis@griffith.edu.au. 


\section{Abstract}

The first synthesis of the fungal natural product (-)-xylariamide $\mathrm{A} \mathbf{1}$ is reported. N,OBis(trimethylsilyl)acetamide induced coupling of D-tyrosine with (E)-but-2-enedioic acid 2,5-dioxo-pyrrolidin-1-yl ester methyl ester 5 produced the dechloro natural product $\mathbf{6}$, which was subsequently monochlorinated using oxone and $\mathrm{KCl}$ to yield synthetic 1 . (-)Xylariamide A 1, (+)-xylariamide A 2 and (-)-dechloroxylariamide A 6 displayed no cytotoxic or antimicrobial activity.

\section{Keywords}

Synthesis; Natural product; (-)-Xylariamide A; (+)-Xylariamide A; (-)-Dechloroxylariamide A; N,O-Bis(trimethylsilyl)acetamide. 
We have recently reported the isolation and structure elucidation of (-)-xylariamide A 1 from the plant-associated microfungus, Xylaria sp. (FRR 5657). ${ }^{1}$ Confirmation of the structure and absolute stereochemistry of 1 resulted from the synthesis of $(+)$-xylariamide A 2. ${ }^{1}$ Both (-)-xylariamide A $\mathbf{1}$ and (+)-xylariamide A $\mathbf{2}$ were screened for toxicity in a brine shrimp (Artemia salina) lethality assay and only the natural product displayed any activity. ${ }^{1}$ Only minute quantities $(0.9 \mathrm{mg})$ of the bioactive natural product 1 were initially isolated from the large-scale fungal fermentation and this prevented more detailed biological evaluations of (-)-xylariamide A. Total synthesis of the chiral chlorinated fungal metabolite $\mathbf{1}$ appeared to be the best means of obtaining quantities of this compound that would allow a more thorough bioactivity profiling. Herein we report a short and efficient synthesis of (-)-xylariamide A 1 along with its cytotoxic and antimicrobial screening results.

Our synthetic approach to $\mathbf{1}$ was based on similar chemistry to that reported for $(+)-$ xylariamide A 2 where the silylating agent $N, O$-bis(trimethylsilyl)acetamide (BSA), was used to form an amide bond between 3-chloro-L-tyrosine and the $N$-succinimide activated ester, (E)-but-2-enedioic acid 2,5-dioxo-pyrrolidin-1-yl ester methyl ester 5. ${ }^{1,2}$ A different synthetic route for the natural product $\mathbf{1}$ had to be designed since we could not find a commercial supplier for 3-chloro-D-tyrosine. Rather than synthesise 3-chloro-D-tyrosine we decided to react 5 with D-tyrosine in the presence of BSA and follow this coupling reaction with the selective monochlorination of the BSA amide product to yield the natural product 1 . The synthesis of (-)-xylariamide A 1 began with the commercially available (E)-but-2enedioic acid dimethyl ester 3 (Scheme 1), selective monohydrolysis of which using aqueous $\mathrm{LiOH}$ in acetone, afforded the previously reported $(E)$-but-2-enedioic acid monomethyl ester $(4,87 \%){ }^{3}$ Coupling 4 with $\mathrm{N}$-hydroxysuccinimide using EDCI in $\mathrm{CH}_{3} \mathrm{CN}$ yielded the known (E)-but-2-enedioic acid 2,5-dioxo-pyrrolidin-1-yl ester methyl ester (5, 40\%). ${ }^{4} \quad(-)$ Dechloroxylariamide A 6 was produced by reacting D-tyrosine, (E)-but-2-enedioic acid 2,5dioxo-pyrrolidin-1-yl ester methyl ester 5 and BSA in DMF at $60{ }^{\circ} \mathrm{C}$ for $16 \mathrm{~h} .{ }^{5}$ Purification was performed using gel permeation chromatography to afford (-)-dechloroxylariamide A (6, $54 \%$ ). The NMR data for 6 were assigned on the basis of 1D and 2D NMR data analysis. Monochlorination of 6 using oxone and $\mathrm{KCl}$ in aqueous $\mathrm{CH}_{3} \mathrm{CN},{ }^{6}$ followed by $\mathrm{C} 18$ HPLC chromatography yielded pure (-)-xylariamide A $(\mathbf{1}, 78 \%)^{7}$ The NMR, MS, UV, IR and $[\alpha]_{\mathrm{D}}$ data for synthetic 1 were identical to those reported for the natural product (-)-xylariamide A $1^{1}$ 
(-)-Xylariamide A 1, the previously synthesised (+)-xylariamide A $\mathbf{2}^{1}$ and (-)dechloroxylariamide A $\mathbf{6}$ were all tested for cytotoxicity against the human cancer cell lines MCF-7 (breast), H460 (non small cell lung), and SF268 (CNS) using the colourimetric sulphorhodamine B assay. ${ }^{8}$ Compounds 1, 2 and 6 showed no cytotoxicity after $72 \mathrm{~h}$ when tested at 5 and $50 \mu \mathrm{g} / \mathrm{mL}$. Compounds 1, 2 and 6 were also tested against a panel of microbial strains known to be associated with nosocomial infection which included multidrug resistant Staphylococcus aureus (wild type MRSA), Staphylococcus aureus (NCCLS 29523), Escherichia coli (ATCC 25922), Enterococcus faecalis (NCCLS 29212), Pseudomonas aeruginosa (ATCC 27853), Streptococcus pyogenes (ATCC 19615), Acinetobacter anitratus (wild type) and Candida albicans (ATCC 60193). Antimicrobial activities were evaluated using a broth micro dilution assay ${ }^{9,10}$ with each compound screened in a double dilution series from 500 to $1.0 \mu \mathrm{g} / \mathrm{mL}$. No microbial growth inhibition was observed at any of these concentrations after $20 \mathrm{~h}$ of dosing.

In conclusion, this paper reports a simple synthesis of the fungal natural product (-)xylariamide A 1 from readily available starting materials. (-)-Xylariamide A 1, (+)xylariamide A 2 and (-)-dechloroxylariamide A 6 displayed no cytotoxic or antimicrobial activity.

\section{Acknowledgements}

R.A.D. acknowledges support provided by a New Researcher Grant from Griffith University. Dr. Jennifer Mitchell from Griffith University is acknowledged for obtaining the HRESIMS data. We thank the Peter MacCallum Cancer Centre, Victoria, Australia for testing compounds $\mathbf{1}, \mathbf{2}$ and $\mathbf{6}$ for cytotoxicity against the panel of human tumour cell lines.

\section{Supplementary data}


${ }^{1} \mathrm{H}$ and ${ }^{13} \mathrm{C}$ NMR spectra and LRESIMS data for compounds $\mathbf{1}$ and 6. Supplementary data associated with this article can be found in the online version.

\section{References and notes}

1. Davis, R. A. J. Nat. Prod. 2005, in press.

2. Patel, V. F.; Andis, S. L.; Kennedy, J. H.; Ray, J. E.; Schultz, R. M. J. Med. Chem. 1999, 42, 2588-2603.

3. The commercial reagent (E)-but-2-enedioic acid dimethyl ester (3,1.44 g, $10 \mathrm{mmol})$ was dissolved in acetone $(70 \mathrm{~mL})$ at $\mathrm{rt}$ and $1 \mathrm{~N}$ aqueous $\mathrm{LiOH}(10 \mathrm{~mL}, 10 \mathrm{mmol})$ was slowly added over 15 min to the stirred solution. The reaction was stirred for $1 \mathrm{~h}$ diluted with $2 \mathrm{~N}$ $\mathrm{HCl}(200 \mathrm{~mL})$, saturated with $\mathrm{NaCl}$ then extracted with EtOAc $(3 \times 200 \mathrm{~mL})$. The EtOAc layer was slowly evaporated and the resulting precipitate was filtered and dried to yield pure (E)-but-2-enedioic acid monomethyl ester $(4,1.13 \mathrm{~g}, 87 \%)$ as a white amorphous solid; mp 142-143 ${ }^{\circ} \mathrm{C}$ (lit. mp $\left.141-141.5^{\circ} \mathrm{C}\right) .^{1,11,12}$

4. (E)-But-2-enedioic acid monomethyl ester (4, $260 \mathrm{mg}, 2 \mathrm{mmol}$ ), EDCI (768 mg, 4 mmol) and $\mathrm{N}$-hydroxysuccinimide $(690 \mathrm{mg}, 6 \mathrm{mmol})$ were dissolved in dry $\mathrm{CH}_{3} \mathrm{CN}(5 \mathrm{~mL})$ and the reaction mixture was stirred at $\mathrm{rt}$ for $24 \mathrm{~h}$. The reaction mixture was pre-absorbed onto silica gel (Alltech 30-40 $\mu \mathrm{m}, 60 \AA$ ) then loaded into a glass column and flushed with $100 \%$ EtOAc $(50 \mathrm{~mL})$. The EtOAc wash was evaporated to dryness, the residue re-dissolved in $100 \%$ DCM and injected onto a MPLC silica (Alltech 30-40 $\mu \mathrm{m}, 60 \AA$ ) packed column (20 $\times 90 \mathrm{~mm}$ ) using isocratic conditions of $40 \%$ EtOAc/60\% hexanes at a flow rate of $6 \mathrm{~mL} / \mathrm{min}$ for $30 \mathrm{~min}$. This yielded pure (E)-but-2-enedioic acid 2,5-dioxo-pyrrolidin-1-yl ester methyl ester $\left(5,180 \mathrm{mg}, 40 \%, t_{\mathrm{R}}=12.0 \mathrm{~min}\right.$ ) as a white amorphous solid; $\mathrm{mp} 93-95{ }^{\circ} \mathrm{C}$ (lit. $\mathrm{mp}$ 93.5-94.5 $\left.{ }^{\circ} \mathrm{C}\right) .{ }^{1,13}$

5. $\quad N, O$-Bis(trimethylsilyl)acetamide $(878 \mu \mathrm{L}, 3.6 \mathrm{mmol})$ was added to (E)-but-2enedioic acid 2,5-dioxo-pyrrolidin-1-yl ester methyl ester (5, $204 \mathrm{mg}, 0.9 \mathrm{mmol})$ and Dtyrosine $(164 \mathrm{mg}, 0.9 \mathrm{mmol})$ in dry DMF $(2 \mathrm{~mL})$ and the reaction was heated at $60{ }^{\circ} \mathrm{C}$ for 16 h. Upon cooling the reaction solution was poured into $2 \mathrm{~N} \mathrm{HCl}(50 \mathrm{~mL})$, saturated with $\mathrm{NaCl}$ then extracted with EtOAc $(2 \times 50 \mathrm{~mL})$. The EtOAc layer was evaporated to dryness under 
reduced pressure to yield a yellow gum (250 mg) which was subsequently dissolved in 100\% $\mathrm{CH}_{3} \mathrm{OH}(2 \mathrm{~mL})$ then loaded onto a Sephadex LH-20 open column $(45 \times 450 \mathrm{~mm})$ and run using $100 \% \mathrm{CH}_{3} \mathrm{OH}$ as eluent at a flow rate of $4.5 \mathrm{~mL} / \mathrm{min}$. All resulting fractions were analysed by TLC and identical fractions combined to yield pure (-)-dechloroxylariamide A (6, $142.6 \mathrm{mg}, 54 \%)$ as a stable clear gum; $[\alpha]^{23}{ }_{\mathrm{D}}-15^{\circ}\left(c 0.270, \mathrm{CH}_{3} \mathrm{OH}\right) ; \mathrm{UV}\left(\mathrm{CH}_{3} \mathrm{OH}\right) \lambda_{\max }$ $(\log \varepsilon) 214$ (4.01), 265 (3.58) nm; IR $v_{\max }(\mathrm{NaCl})$ 3600-3100, 1720, 1664, 1546, 1516, 1445, 1349, 1308, 1272, 1232, 1198, 1173, 1113, 1024, 979, 832, $766 \mathrm{~cm}^{-1} ;{ }^{1} \mathrm{H}$ NMR (500 MHz, DMSO-d $\left.{ }_{6}\right) \delta 2.76(1 \mathrm{H}, \mathrm{dd}, J=14.0,9.5 \mathrm{~Hz}, \mathrm{H}-7 \mathrm{a}), 2.98(1 \mathrm{H}, \mathrm{dd}, J=14.0,5.0 \mathrm{~Hz}, \mathrm{H}-7 \mathrm{~b})$, $3.71\left(3 \mathrm{H}, \mathrm{s}, 13-\mathrm{OCH}_{3}\right), 4.44(1 \mathrm{H}, \mathrm{ddd}, J=9.5,7.5,5.0 \mathrm{~Hz}, \mathrm{H}-8), 6.53(1 \mathrm{H}, \mathrm{d}, J=15.5 \mathrm{~Hz}, \mathrm{H}-$ 12), $6.64(2 \mathrm{H}, \mathrm{d}, J=8.5 \mathrm{~Hz}, \mathrm{H}-3, \mathrm{H}-5), 7.00(2 \mathrm{H}, \mathrm{d}, J=8.5 \mathrm{~Hz}, \mathrm{H}-2, \mathrm{H}-6), 7.06$ (1H, d, $J=$ $15.5 \mathrm{~Hz}, \mathrm{H}-11), 8.79$ (1H, d, $J=7.5 \mathrm{~Hz}, 8-\mathrm{NH}), 9.18$ (1H, brs, 4-OH), 12.76 (1H, brs, 9-OH); ${ }^{13} \mathrm{C}$ NMR (125 MHz, DMSO-d $) \delta 35.9$ (C-7), 51.9 (13- $\left.-\mathrm{OCH}_{3}\right), 54.1$ (C-8), 115.0 (2C, C-3, C-5), 127.3 (C-1), 128.5 (C-12), 129.9 (2C, C-2, C-6), 137.1 (C-11), 155.9 (C-4), 162.6 (C10), 165.4 (C-13), 172.5 (C-9); (-)-LRESIMS m/z (rel. int.) 134 (10), 216 (5), 248 (5), 278 (5), 292 (100); (-)-HRESIMS m/z $292.08401\left(\mathrm{C}_{14} \mathrm{H}_{14} \mathrm{NO}_{6}[\mathrm{M}-\mathrm{H}]^{-}\right.$requires 292.08268).

6. Ghosh, A. K.; Swanson, L. J. Org. Chem. 2003, 68, 9823-9826.

7. Oxone (105 mg), $0.171 \mathrm{mmol})$ was added to (-)-dechloroxylariamide A (6, $50 \mathrm{mg}$, $0.171 \mathrm{mmol})$ in $\mathrm{CH}_{3} \mathrm{CN}(5 \mathrm{~mL})$ and $\mathrm{H}_{2} \mathrm{O}(15 \mathrm{~mL})$ then $\mathrm{KCl}(20 \mathrm{mg}, 0.257 \mathrm{mmol})$ was added in two equal portions over $1 \mathrm{~h}$ and the reaction mixture was stirred at $\mathrm{rt}$ for $72 \mathrm{~h}$, then poured into $2 \mathrm{~N} \mathrm{HCl}(50 \mathrm{~mL})$, saturated with $\mathrm{NaCl}$ then extracted with EtOAc $(2 \times 50 \mathrm{~mL})$. The EtOAc layer was evaporated to dryness under reduced pressure to yield a yellow gum (76 $\mathrm{mg})$. This material was dissolved in DMSO $(750 \mu \mathrm{L})$ and $\mathrm{CH}_{3} \mathrm{OH}(250 \mu \mathrm{L})$ and purified by preparative HPLC on a Thermo Hypersil C18 BDS $5 \mu \mathrm{m} 143 \AA$ column $(21.2 \times 150 \mathrm{~mm})$ using isocratic conditions of $40 \% \mathrm{CH}_{3} \mathrm{OH} / 60 \%$ aqueous TFA $(0.2 \%)$ at a flow rate of 6 $\mathrm{mL} / \mathrm{min}$. This yielded pure (-)-xylariamide A (1, $43 \mathrm{mg}, 78 \%)$ as a stable clear gum; $[\alpha]^{23}{ }_{\mathrm{D}}$ $-16^{\circ}$ (c $\left.0.250, \mathrm{CH}_{3} \mathrm{OH}\right)\left(\right.$ lit. $\left.[\alpha]^{24}{ }_{\mathrm{D}}-22^{\circ}\left(\mathrm{c} 0.060, \mathrm{CH}_{3} \mathrm{OH}\right)\right) ;^{1} \mathrm{UV}\left(\mathrm{CH}_{3} \mathrm{OH}\right) \lambda_{\max }(\log \varepsilon) 208$ (4.01), $220 \mathrm{sh}$ (3.93), 276 (3.36) nm; IR $v_{\max }(\mathrm{NaCl}) 3500-3200,1712,1665,1549,1512$, 1440, 1345, 1294, 1237, 1196, 1173, 1057, 1024, 976, 824, 766, $667 \mathrm{~cm}^{-1}$; ${ }^{1} \mathrm{H}$ NMR (500 MHz, DMSO-d $\left.{ }_{6}\right) \delta 2.78(1 \mathrm{H}, \mathrm{dd}, J=14.0,9.0 \mathrm{~Hz}, \mathrm{H}-7 \mathrm{a}), 2.99(1 \mathrm{H}, \mathrm{dd}, J=14.0,5.0 \mathrm{~Hz}, \mathrm{H}-$ 7b), $3.71\left(3 \mathrm{H}, \mathrm{s}, 13-\mathrm{OCH}_{3}\right), 4.46(1 \mathrm{H}$, ddd, $J=9.0,8.5,5.0 \mathrm{~Hz}, \mathrm{H}-8), 6.54(1 \mathrm{H}, \mathrm{d}, J=15.5$ Hz, H-12), 6.84 (1H, d, $J=8.5$ Hz, H-5), 6.97 (1H, dd, $J=8.5,1.5$ Hz, H-6), 7.05 (1H, d, $J=$ 
15.5 Hz, H-11), 7.17 (1H, d, $J=1.5 \mathrm{~Hz}, \mathrm{H}-2), 8.80$ (1H, d, $J=8.5 \mathrm{~Hz}, 8-\mathrm{NH}), 9.94$ (1H, brs, 4-OH), 12.82 (1H, brs, 9-OH), ${ }^{13} \mathrm{C}$ NMR (125 MHz, DMSO-d 6 ) $\delta 35.5$ (C-7), 52.0 (13$\left.\mathrm{OCH}_{3}\right), 53.8$ (C-8), 116.4 (C-5), 119.1 (C-3), 128.6 (2C, C-6, C-12), 128.9 (C-1), 130.2 (C2), 136.9 (C-11), 151.6 (C-4), 162.7 (C-10), 165.4 (C-13), 172.3 (C-9); (-)-LRESIMS m/z (rel. int.) 168 (20), 170 (7), 250 (10), 252 (3), 282 (10), 284 (3), 312 (10), 314 (3), 326 (100), 328 (33); (-)-HRESIMS m/z $326.04252\left(\mathrm{C}_{14} \mathrm{H}_{13} \mathrm{NO}_{6}{ }^{35} \mathrm{Cl}[\mathrm{M}-\mathrm{H}]^{-}\right.$requires 326.04369).

8. Skehan, P.; Storeng, R.; Scudiero, D.; Monks, A.; McMahon, J.; Vistica, D.; Warren, J. T.; Bokesch, H.; Kenney, S.; Boyd, M. R. J. Natl. Cancer Inst. 1990, 82, 1107-1112.

9. Jorgensen, J.H.; Weigel, L.M.; Swenson, J.M.; Whitney, C.G.; Ferraro, M.J.; Tenover, F.C. Antimicrob. Agents Chemother. 2000, 44, 2962-2968.

10. Lister, P. D. Antimicrob. Agents Chemother. 2002, 46, 69-74.

11. Niwayama, S. J. Org. Chem. 2000, 65, 5834-5836.

12. Dymicky, M.; Buchanan, R. L. Org. Prep. Proced. Int. 1985, 17, 121-131.

13. Andruszkiewicz, R.; Chmara, H.; Milewski, S.; Borowski, E. Int. J. Pept. Protein Res. 1986, 27, 449-453. 
<smiles>COC(=O)/C=C/C(=O)N[C@@H](Cc1ccc(O)c(Cl)c1)C(=O)O</smiles>

(-)-Xylariamide A 1<smiles>COC(=O)/C=C/C(=O)NC(Cc1ccc(O)c(Cl)c1)C(=O)O</smiles>

(+)-Xylariamide A 2

Figure 1. Structures for 1 and 2.<smiles>[R20]C(=O)/C=C/C(=O)OC</smiles>

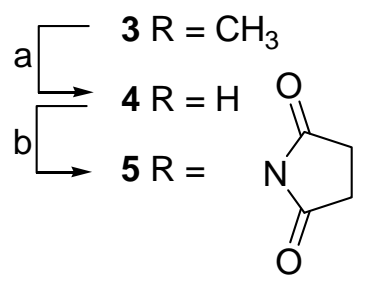

(-)-Xylariamide A 1

Scheme 1. Total synthesis of (-)-xylariamide A 1. Reagents and conditions: (a) LiOH, acetone, rt, $1 \mathrm{~h} \mathrm{(87 \% );}$ (b) $\mathrm{N}$-Hydroxysuccinimide, $\mathrm{EDCl}, \mathrm{CH}_{3} \mathrm{CN}, \mathrm{rt}, 24 \mathrm{~h}$ (40\%); (c) D-tyrosine, BSA, DMF, $60^{\circ} \mathrm{C}, 16 \mathrm{~h}(54 \%)$;

(d) Oxone, $\mathrm{KCl}, \mathrm{CH}_{3} \mathrm{CN} / \mathrm{H}_{2} \mathrm{O}$, rt, $72 \mathrm{~h}(78 \%)$. 


\section{Synthesis of the fungal natural product (-)-xylariamide A}

Rohan A. Davis* and Michael Kotiw

The first synthesis of the fungal natural product (-)-xylariamide A $\mathbf{1}$ is reported. $\mathrm{N}, \mathrm{O}$-Bis(trimethylsilyl)acetamide induced coupling of D-tyrosine with (E)-but-2-

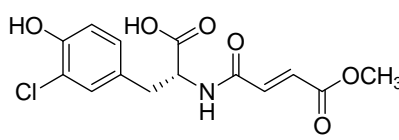

(-)-Xylariamide A 1 enedioic acid 2,5-dioxo-pyrrolidin-1-yl ester methyl ester 5 produced the dechloro natural product $\mathbf{6}$, which was subsequently monochlorinated using oxone and $\mathrm{KCl}$ to yield synthetic 1. (-)-Xylariamide A 1, (+)-xylariamide A 2 and (-)dechloroxylariamide A $\mathbf{6}$ displayed no cytotoxic or antimicrobial activity. 